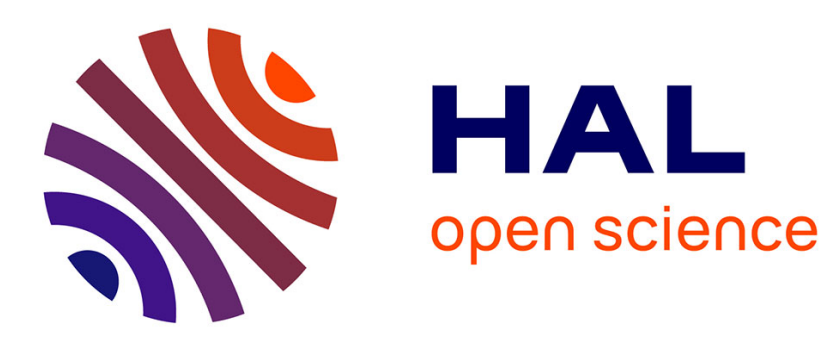

\title{
A risk-based approach to drive conceptual design taking into account low-maturity products
}

\author{
Mehdi El Amine, Nicolas Perry, Jerome Pailhes
}

\section{To cite this version:}

Mehdi El Amine, Nicolas Perry, Jerome Pailhes. A risk-based approach to drive conceptual design taking into account low-maturity products. International Journal on Interactive Design and Manufacturing, 2016, 10.1007/s12008-016-0354-z . hal-01500956

\section{HAL Id: hal-01500956 \\ https://hal.science/hal-01500956}

Submitted on 6 Apr 2017

HAL is a multi-disciplinary open access archive for the deposit and dissemination of scientific research documents, whether they are published or not. The documents may come from teaching and research institutions in France or abroad, or from public or private research centers.
L'archive ouverte pluridisciplinaire HAL, est destinée au dépôt et à la diffusion de documents scientifiques de niveau recherche, publiés ou non, émanant des établissements d'enseignement et de recherche français ou étrangers, des laboratoires publics ou privés. 


\title{
A risk-based approach to drive conceptual design taking into account low-maturity products
}

\author{
Medhi El Amine ${ }^{1,}$ Nicolas Perry ${ }^{2}$, Jérôme Pailhès ${ }^{2}$
}

1.Laboratoire d'Innovation Durable et de Recherche Appliquée (LIDRA)UniversiapolisAgadirMorocco

2.Arts et Métiers ParisTechTalenceFrance

Résumé The presence of immature concepts makes difficult decision making at upstream phase of a development project. Rework tasks in collaborative development projects dealing with immature design concepts are very frequent and are responsible of cost overruns and schedule delays. In addition, decisions taken during conceptual design stage have the most important influence on product life cycle cost. In this context, a useful practice is to develop two or more concepts in parallel and delay decision making. Even so, a first screening of concepts is usually needed because of the substantial resources and time required in development phase. In this paper, an approach of concepts screening is proposed at conceptual design phase on the basis of two metrics. The first assesses ability of concepts to meet requirements with integration of maturity. Fuzzy logic tools are used to capture and propagate imprecision in embodiment design choices and behavior models. The second metric aims to assess expected performance of novel concepts compared with an existent product. It uses generalized ordered weighted averaging (GOWA) operator. A parametric aggregation function is also proposed as a support when using the two metrics. The proposed approach was applied to the development of a solar collector.

Keywords Decision making · Novel concept - Set-based design · Maturity · Conceptual design $\cdot$ Performance $\cdot$ Trade-off

\section{Introduction}

In a broad sense, the ability of decision makers to choose the best concept in engineering design is strongly conditioned by two factors : (i) having a clear definition of design objectives, and (ii) being able to evaluate or predict the performance of initial design concepts. In practice, it is very difficult to 
predict accurately the performance of novel and immature concepts and design objectives are usually vague and imprecise in the early phases of a development project. Paradoxically, life-cycle cost can be impacted up to $70 \%$ by decisions taken during conceptual design phase [1]. The challenge is how to support the decisions that are made in light of uncertainty and ambiguity.

In order to minimize risk and reduce the cost of regret in later development stages, many design departments adopt the approach called parallel set narrowing process $[2,3]$ which consists of developing several concepts in parallel until enough knowledge is acquired to facilitate the selection of the most appropriate one in regard of design requirements (Fig. 1). Although developing many concepts is effective in minimizing risk, the usefulness of such a practice is limited by the substantial cost and time required for the development activities. Eliminating operations are thus required to reduce the initial set of proposed concepts such that it can be supported by company resources and allocated budgets.

Many authors recognize the importance of assessing and integrating maturity in decision making [4-6]. Within our context, concept maturity is defined according to Dunn [7] : "Maturity makes possible a "linear" design process, each stage flows sequentially - no backtracking. Non-maturity results in an "orbital process", a circular sequence of repetition, the total time taken being several times that for the linear process". According to this definition, a concept becomes more mature when it requires fewer modifications. Indeed, once a concept is chosen to be developed by the company, designers start embodiment design stage which consists in rough arrangements and selections of structural dimensions, materials, components and technologies [4]. For an immature concept, these choices are made with insufficient knowledge and, as a result, the required performance is usually not met. The impact of concept maturity is thus closely related to initial requirements. Some authors even define maturity as the association of knowledge and performance [8]. As shown in Fig. 1, the progress of concept development is accompanied by a narrowing in the set of possible embodiment choices combinations (finite elements analysis, mechanical tests) until the most suitable combination of these choices is found. The clarification of design objectives also accelerates the narrowing process as shown in Fig. 1. Concept maturity is thus increasing. Apart from the risk of non-completion of design requirements, cost overruns and schedule delays are very frequent when developing immature concepts [9]. This aspect should be taken into account when selecting concepts. It is highly recommended that companies have a concept with a high degree of maturity $[6,9]$. On the other hand, the desire to be distinguished from the competition and to keep pace with a rapidly changing market, means that decision-makers are encouraged to adopt an innovation strategy by developing novel concepts. Market competitiveness through innovation is the common strategy of developed companies. In addition, the companys long-term sustainability may depend on its innovation strategy [10]. In summary, the choice of the set of concepts to develop in the context of SBCD is driven by both the need for performance improvement and failure risk limitation. 


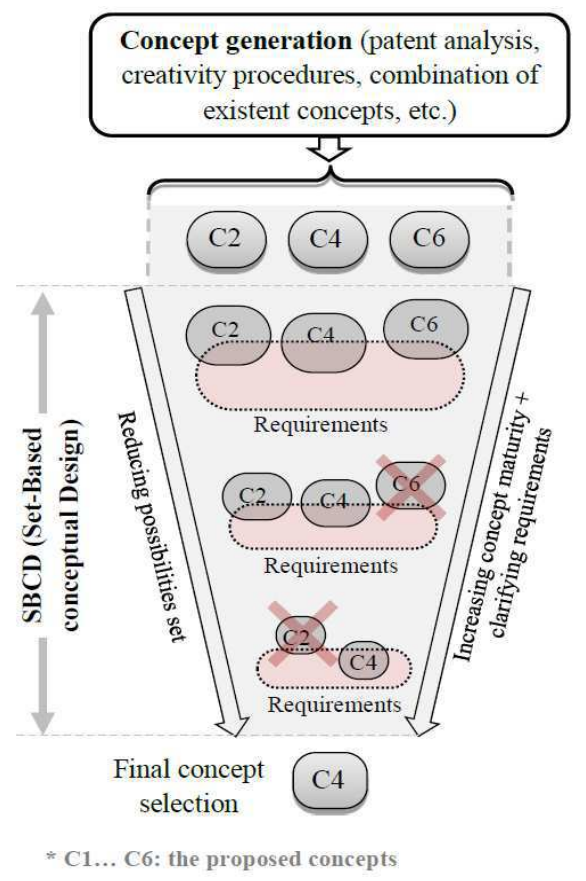

Figure 1 Schematization of global development process in the context of set-based design

\section{Background}

2.1 Decision making process in engineering design

Generally speaking, the procedure of decision making in engineering design is composed of four steps cited below $[11,12]$. The error in final decision making is due to the association of imprecisions occurring in each of the four steps (Fig. 2 ). Understanding these decision making process and imprecisions occurring in each step is a first step for an effective integration of concept maturity degree.

The definition of a solution. When a concept is proposed, it is usually necessary to define a concrete solution by fixing embodiment design choices (rough arrangements and selections of structural dimensions, materials, components and technologies). For a concept with low maturity, designers do not have sufficient knowledge to define the wright combination of these choices. Many approximations and hypothesizes are thus usually adopted. These can be a source of errors in the evaluation of the concept.

The observation step. Data are generated about each concept (mass, maximum stress, carbon footprint, etc.) and are required to assess the satisfaction of each criterion. In this work, they are noted OV (observation variables). 


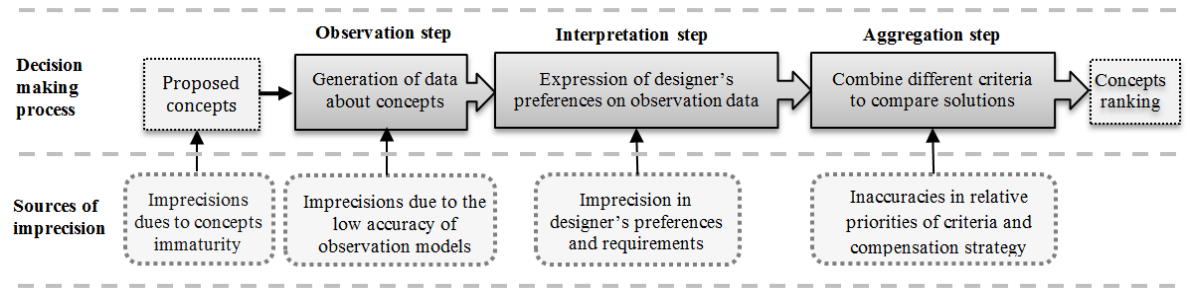

Figure 2 Decision-making process in engineering design

These variables are evaluated using behavior models (finite element models, expert judgments, mechanical tests, historic data, etc.) that can be inaccurate and thus induce imprecision in estimated values.

The interpretation step. Designers express their preferences for each design criterion on the basis on data collected in previous step (observation variables). Designers preferences and requirements can also contain imprecision. The procedure of interpretation is normalized in our study. For each criterion, an interpretation variable (noted IV) between 0 and 1 is affected to express design satisfaction degree.

The aggregation step. Designers combine IV for each design criterion in order to determine the best alternative(s). In this step, imprecision can affect criteria relative priority and compensation degree between criteria.

In literature many multi-criteria decision aid (MCDA) method are proposed. In this work, we consider only those specific to engineering design problems. In this framework, a recognized method is that of Method of Imprecision (MoI) proposed by Wood and Antonsson [13]. It allows designers to formalize impression in concept evaluation using fuzzy logic [14]. Each concept is then evaluated using several preference values which are finally aggregated into a generalized preference. The MoI proposes axioms to provide a framework for application to the field of engineering design, and the ability to use different strategies to express the will of designers. However, when evaluating concepts with different maturity degree, two main limits can be detected : (i) the axiom of annihilation leads to a total discrimination to concepts that does not satisfy one of criteria, whereas for an immature concept. (ii) The evaluation of concepts is based on a unique indicator which leads to no distinction between concepts of different maturity degree, whereas an immature concept presents a real risk for the company.

2.2 Concept selection methods in literature

Various concept selection methods exist in the literature. Choosing the most appropriate method is itself a critical decision as different methods can 
yield different outcomes for the same problem $[11,15,16]$. The choice of the most appropriate method is very dependent on the decision making situation [17]. The decision-maker must be aware of the underlying principle of the chosen method and verify that it is in accordance with his own decision-making situation. The state of the art proposed in this section aims to verify that the concept selection methods proposed in the literature are appropriate for our decision-making process.

Multi-Attribute Utility Theory (MAUT) is one of the most recognized and accepted methods in decision making. It was initially proposed by Neumann and Morgenstern [18] for economic decision-making. Pahl and Beitz [19] were the first to incorporate MAUT into a systematic engineering design process. They suggest six steps for applying MAUT in engineering design : (i) design criteria are first established (such as cost, resistance, durability, etc.), (ii) these criteria are rated on a scale of 0-100 such that the sum of the weights for all criteria is equal to 100 , (iii) evaluation parameters are then defined to enable quantification of concept comparisons with one another (This corresponds to step 1 in Fig. 2), (iv) scores are assigned as a utility to each of the evaluation parameters (This corresponds to step 2 in Fig. 2), (v) utility theory is used to calculate the concept value, which is the product of the criterion parameter score and the criterion weighting (This corresponds to step 3 in Fig. 2), (vi) concepts are ranked based on overall utility and the concept with the highest utility score is selected. According to Scott and Antonsson [20], the use of a weighted sum in engineering design is not appropriate since the aspect of decision-making cannot be compensated, contrary to decision in the field of economics, where all aspects of decision can always be translated into associated cost. He proposed a set of mathematical axioms that must be respected by an aggregation operator when dealing with an engineering design decision.

Pahl and Beitzs [19] utility theory does not directly accommodate the analysis of imprecisely characterized alternatives. By extending uncertainty representations to model imprecision explicitly, it is possible to apply the principles of utility theory to such problems. This can be done by using discrete/continuous intervals or fuzzy logic. The expected utility for a given concept is thus not known precisely and becomes an interval or fuzzy representation. However, this can lead to situations of indeterminacy [21], meaning that the decision-makers have no rational basis for choosing one concept over another.

Based on a fuzzy representation of expected concept utilities (as shown in Fig. 3), Roubens [22] proposed the metric of weak dominance than can be interpreted, when applied to a given concept $\mathrm{A}$, as the truth value of the statement "Concept A is better than X, for every concept X in the set of proposed concepts". By producing a single value of concept evaluation, it eliminates the problem of indeterminacy and thus facilitates the selection of a concept. However, it does not assess risk and expected utility separately since each design concept is assessed using single numerical attribute.

Technology Readiness Level (TRL), a 9-level ordinal scale, related to a 9-level semantic scale [23], is a widely used metric in the aeronautics, aeros- 
pace and weapons industry to assess maturity of developed concepts. It allows decision-makers to ensure that a concept has enough maturity to be introduced into the system. Conrow [24] argues that mathematical operations performed on TRL metrics can lead to systematic errors because of their ordinal nature. He proposes a calibration of the TRL scale based on an analytic hierarchical process (AHP) that results in estimated cardinal values being assigned to each TRL scale level, allowing for mathematical analysis using TRL as an independent variable. It has therefore become possible to aggregate it with other metrics. The state of progress of an innovative technology (that is defined by TRL) can be linked to eliminating imprecision in design definition and a concept with a higher TRL score has a better chance of being feasible. However, TRL remains a coarse metric for the maturity of the concept. It does not allow for a fine assessment on the state of imprecision in design definition.

\section{Proposed approach}

As explained in introduction, when decision makers are establishing the set of concepts to develop for downstream phases of development process, their choices are driven by two different and complementary tendencies. On the one hand, they want to obtain rapidly a feasible concept with high maturity degree in order to reduce risk. On the other hand, they wish to develop novel concepts that elicit a higher performance than existing products. The essence of the proposed approach is to propose two numerical metrics that represent these two tendencies. They can constitute an understandable and useful support for decision makers when choosing concepts to develop. The procedure of their calculation is described in this section. A parametric aggregation function is also proposed at the end of this section to allow decision makers to establish the set of concepts to develop with the desired trade-off between the two metrics. The entire approach is illustrated in Fig 3.

\subsection{Required data}

In order to use the proposed metric (the ability to satisfy requirement and concept performance), a set of preliminary data is required. Design criteria are first established with the acceptance threshold for each one depending on decision makers needs. The annihilation axiom is specific to engineering design $[13,25,26]$ and is considered in our approach. It states that if the satisfaction for any one criterion of the proposed design is null (acceptance thresholds not reached) then the design is not valid regardless the satisfaction degree of the other criteria.

Weights are then assigned to criteria to express their relative importance degrees. They are denoted wj (for a criterion j). Given the heterogeneous nature of the criteria, it is difficult and risky to directly assign weights to criteria. In our work, the analytical hierarchy process (AHP) was used to define 


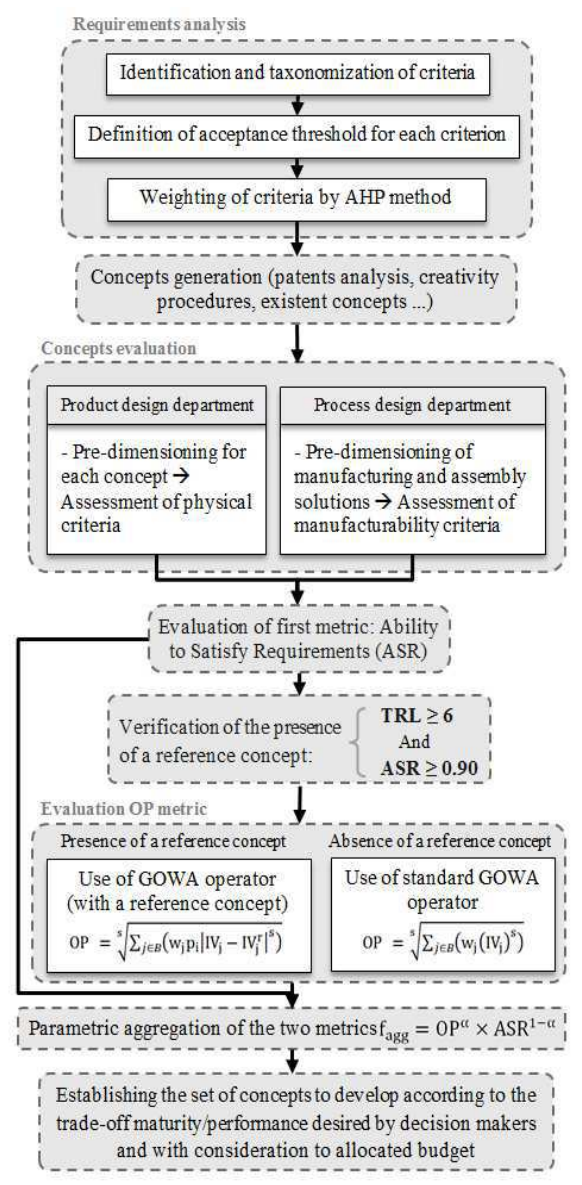

Figure 3 Steps of the proposed approach

these weights. The use of pairwise comparison is more appropriate to deal with criteria of heterogeneous natures [11]. Another advantage of the AHP is the consideration of the hierarchical decomposition of criteria. However, this method is subject to a consistency constraint. In order to limit the inconsistencies that occur when performing pairwise comparisons, the Consistency Ratio (CR) proposed by Saaty [27] was used as a guidance to check for consistency. As recommended by Saaty [27], a value of 0.1 was used as threshold for CR.

The set of possible concepts is then generated. This can be achieved by patent analysis, creativity procedures, analysis of existent concepts from previous projects/competitors, etc.

Finally the pre-dimensioning of the proposed concepts is performed by the design team. Indeed, the evaluation of a concept usually requires the definition of a concrete solution. It means that embodiment design choices such as rough arrangements and selections of structural dimensions, materials, components 
and technologies must be fixed. Since the product design is strongly driven by manufacturing requirements (Fig 7), the pre-dimensioning of manufacturing process is also performed in order to evaluate manufacturability criteria. OV (observation variables) are then generated as a result of this step. This data are then converted to IV (interpretation values) in accordance with decision makers preferences and requirements.

3.2 First metric : the Ability to Satisfy Requirements (ASR)

In the previous step, a first architecture of product is established by fixing the different choices in embodiment design. This allows designers to evaluate the proposed concept against each criterion, including manufacturability criteria. For an immature concept, designers do not have sound basis when fixing these choices and, as a result, one or more criteria are often not satisfied. In our formulation of the problem it means that one or more OVs do not reach the corresponding acceptance threshold. It results in a zero IV. In practice, designers do not systematically reject the concept. They consider the fact that modifications could eventually be introduced in embodiment design choices to insure the satisfaction of these criteria. It usually takes many iterations of trial-error to find the right combination of embodiment design choices. This procedure can be very time consuming when many concepts are proposed. In a broad sense, the proposed maturity metric, denoted $A S R$ (Ability to Satisfy Requirements), evaluates the opportunity of reaching design requirements by exploring the set of possible solutions in embodiment design. The steps of its calculation are described in this paragraph. An illustrative example is shown in Fig 4. It represents the case of non-achievement of requirement on maximum angular deformation of the truss concept.

Only unsatisfied criteria are considered in the evaluation the $A S R$. For each of these criteria (denoted $\mathrm{j}$ ), a maturity index related to this criterion is evaluated and denoted $A S R_{j}$. A four-step procedure is used to evaluate this index :

Step (i) Identify embodiment design choices (structural dimensions, materials, components and technologies) that have a significant impact on the OV considered. This task can be done by the association of experience/intuition of designers and the behaviors models used in the pre-dimensioning step.

Step (ii) Describe the level of imprecision in these embodiment design choices. As mentioned before, the low maturity of a concept is manifested by the imprecision when making a choice for an embodiment design element. This step consists of describing this imprecision by identifying the remaining possible choices that designers are unable to settle. 


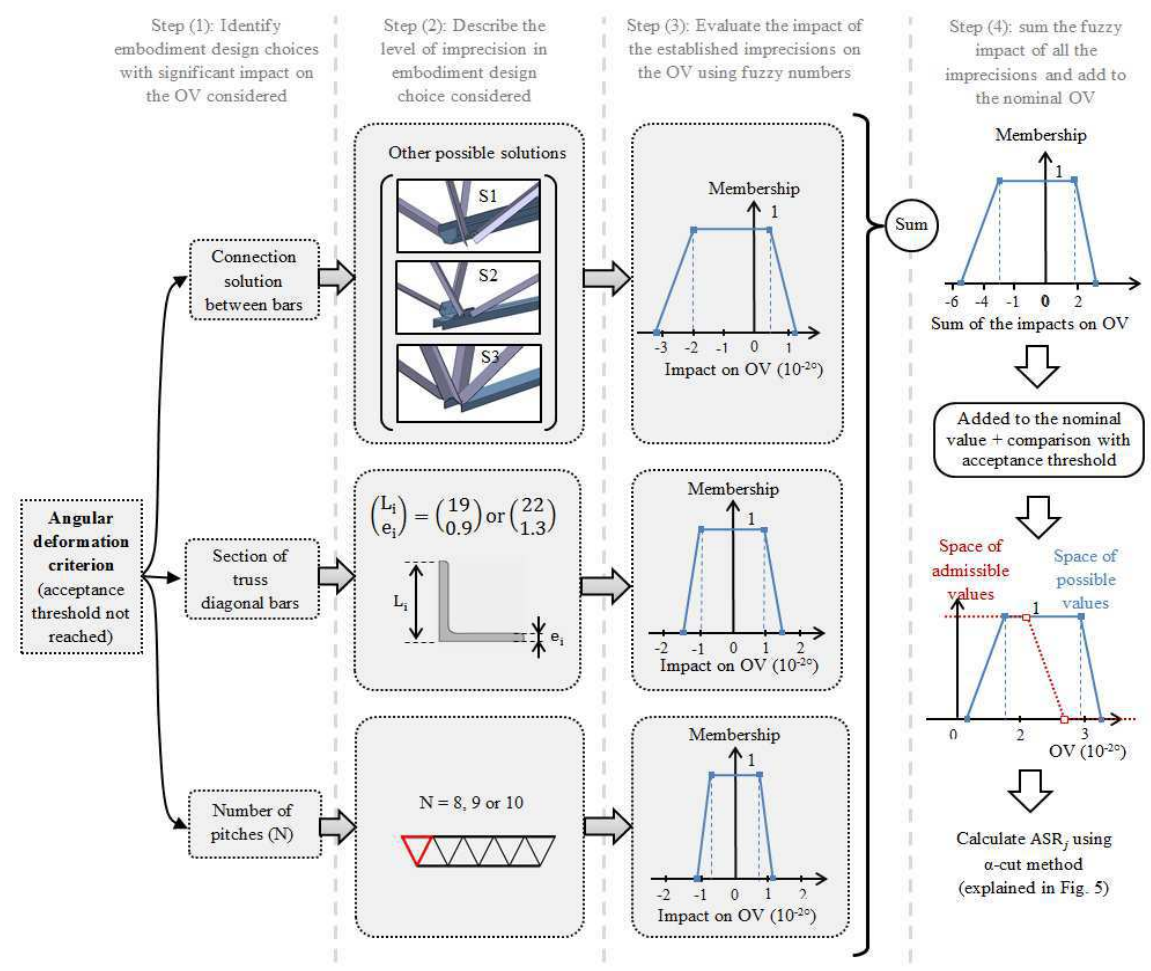

Figure 4 Illustrative example of ASR calculation procedure

Step (iii) Evaluate the impact of the established imprecisions on the OV using fuzzy numbers. This consists of evaluating of changing the embodiment design element (in the set of possible choices) on the on the OV estimated at pre-dimensioning step. This can be performed using behavior models used at the pre dimensioning step. Since the used model can very rough at this design stage, fuzzy numbers are used to capture designers imprecision when evaluating the impacts. It concerns immaturity of manufacturing process, imprecision in behavior models and, imprecision in embodiment design choices.

Step (iv) Sum the fuzzy impacts of all the imprecisions and add to the nominal $O V$. The different imprecision impacts are summed together to obtain a total impact on OV. This quantity is then added to the nominal value of the OV that was evaluated in pre-dimensioning step. After that, $A S R_{j}$ of the criterion $\mathrm{j}$ is calculated as the ratio between the overlap surface (intersection between possible values of $\mathrm{OV}$ and admissible values) and the possible values. This overlap region is graphically represented in Fig 5 . The -cut method is used to calculate this value and explained below. 


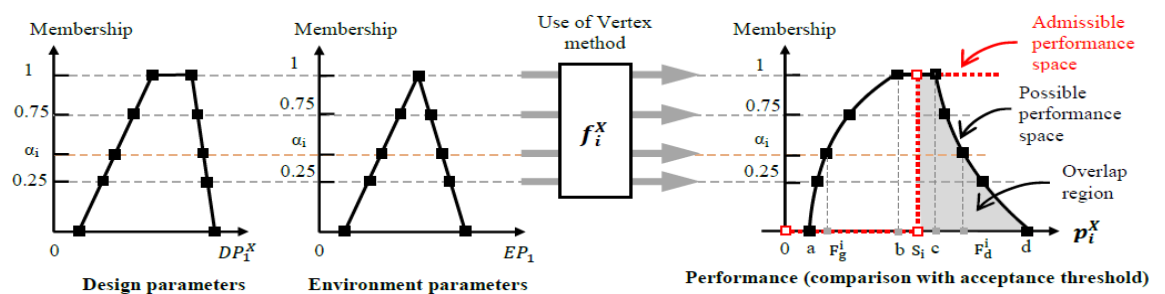

Figure 5 Using the Vertex method to map design space to performance space (case of one design parameter and one environment parameter)

$$
\begin{gathered}
A S R_{j}=\frac{1}{N} \sum_{i=1}^{N} C^{i} \\
x=\left\{\begin{array}{l}
0 \text { if } S^{i}<F_{g}^{i} \\
\frac{S^{i}-F_{g}^{i}}{F_{d}^{i}-F_{g}^{i}} \text { if } F_{g}^{i}<S^{i} \leq F_{d}^{i} \\
1 \text { if } S^{i} \geq F_{g}^{i}
\end{array}\right. \\
\left\{\begin{array}{l}
F_{g}^{i}=a+\frac{i}{N} \times(b-a) \\
F_{d}^{i}=c+\frac{i}{N} \times(d-c) \\
S^{i}=S_{g}+\frac{i}{N} \times\left(S_{d}-S_{g}\right)
\end{array}\right.
\end{gathered}
$$

The final maturity index is obtained by the multiplication of all $A S R_{i}$ as shown in Equation 5. The product was used since the annihilation axiom is adopted in our case. Achieving acceptance threshold for each criterion is a required condition for the acceptance of the concept.

$$
A S R=\prod_{i} A S R_{i}
$$

As the development progress, concept maturity increases and many infeasible combinations are progressively eliminated. The proposed procedure offers a simple but also a rough way to evaluate the opportunity of improving an OV related to unsatisfied or pseudo-satisfied criteria. The fuzzy numbers and related arithmetic are a powerful tool to capture and propagate imprecision related to product definition and also designer estimations. Two main considerations are not included within this approach : the possible synergies between two or more embodiment design features when they are changed together and, the impact of an embodiment design feature on the other criteria when its changed. However, we argue that these data are difficult to obtain at very upstream 
step of product development process. More advanced development tasks (later development steps) are required for this.

In many industries, design department need to fix a maturity threshold for product with critical role in the global system $[9,28,29]$. It allows the different stakeholders to know whether the concepts of the program have acceptable levels of risk. The department of defense for example fixes a threshold of $T R L=6$ [28]. In our approach, this threshold is defined by two conditions : (i) the ASR of this concept must be higher than 0.9 (ii) a prototype of the concept must be tested in a relevant environment (equivalent to $T R L=6$ ) as a proof of the validity of behaviors models. In this paper, a concept verifying these conditions is called Reference concept.

\subsection{Second metric : Overall Performance (OP)}

The ASR metric proposed in the previous paragraph is representative of the confidence given to a concept since it measures the opportunity of reaching acceptance thresholds of unsatisfied criteria. However, this is insufficient when screening concept. Decision making should have a more global view about concept performance by taking into account also the strengths of concept.

Otherwise, the question that decision makers need to consider is : if the decision is made to invest resources and time in development activities to settle unsatisfied criteria, how much the global performance of the concept could be. The question is especially pertinent when a pre-existing concept exist which has already been developed and tested.

The OV are transformed into IV which are dimensionless variable between 0 and 1 . It represents the desirability degree for a given OV. The OP metric is obtained by the aggregation of IV using generalized ordered weighted averaging (GOWA) operator (equation 6) proposed by Yager [30]. This function was chosen since a multitude of compensation degree between criteria can be used by varying the parameter s between -10 and 10 .

$$
O P=\sqrt[s]{\sum_{j}^{n}\left(I V_{j}\right)^{2}}
$$

$j$ is the criterion index. $w_{j}$ is the weight of criterion $j . \mathrm{n}$ is the number of criteria.

In many case of development projects, company has a mature concept that result from previous development project.

In this case, the interest of developing a novel concept is to realize a better performance. It is thus appropriate that the aggregation function considers the reference concept in determining the performance of the new concepts. The equation (6) is thus modified to use the distance from the reference concept. 
The expression becomes :

$$
O P_{r}=\sqrt[S]{\sum_{j}^{n} w_{j} p_{i}\left|I V_{j}-I V_{j}^{r}\right|^{S}}
$$

$I V_{j}^{r}$ is the $I V$ of criterion $\mathrm{j}$ for the reference concept. pi is a parameter introduced to take into account the sense of the improvement. $p_{i}=1$ when the improvement is positive and $p_{i}=-1$ when the improvement is negative.

\subsection{Aggregation of the two metrics}

Once the two metrics are estimated $(A S R$ and $O P$ ), the decision makers can choose the appropriate concepts by applying the desired trade-off between the confidence aspect (represented by ASR metric) and the performance aspect (represented by OP metric). Here, since only two metrics are treated, the decision maker can do without the use of an aggregative function; the decision maker is able to aggregate intuitively both metrics. However, an aggregation function is provided to constitute a further support to the decision makers. This aggregation function is given in the Equation 8 . The weighed product was used since it yields a zero score when $A S R$ or $O P$ is null.

$$
f_{\text {agg }}=O P^{\alpha} \times A S R^{1-\alpha}
$$

is a parameter introduced to adjust the trade-off level desired by decision makers. When establishing the set of concepts to develop. We argue that the most suitable case when constructing the set of concepts to develop is take values from different regions along the interval $[0,1]$. In this way, the constructed set of concepts will contain mature concepts (high $A S R$ ) that minimize risk and innovative concept that highlight a better performance (high $O P$ ). The choice of the set of concepts to develop must also take into account the planned budget for the development project.

In the next section, the proposed methodology is applied to an example from a real product development project. The objective is to measure the usefulness of the methodology in our industrial case.

\section{Application on industrial case}

Compared to photovoltaic panel, the major advantage of Concentrating Solar Power (CSP) is the possibility of storing thermal energy which allows the production electricity without interruption. The component studied in our case is the solar collector. Its main function is to concentrate and redirect sunlight onto absorber tubes to heat up the working fluid. The recovered heat is then used to generate high pressure steam which drives a turbine in order to produce electricity. The solar collector is composed of a reflecting surface and a metal structure, whose function is to give and maintain reflecting glass shape 


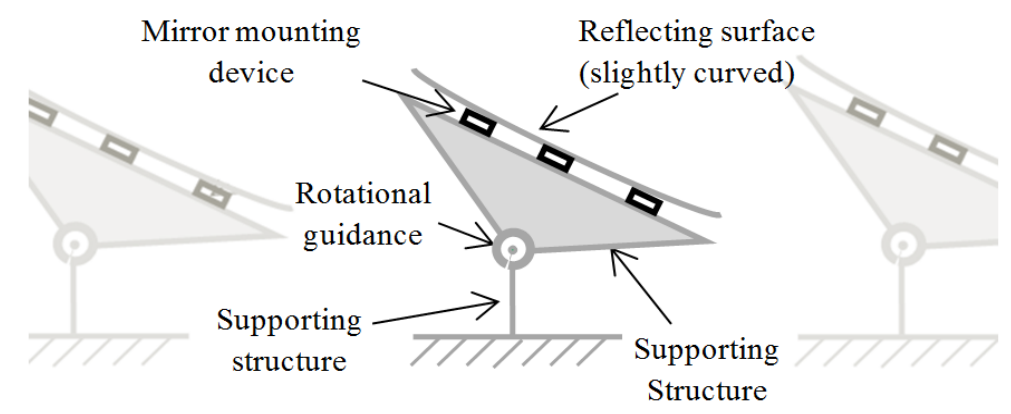

Figure 6 Schematization of solar collector

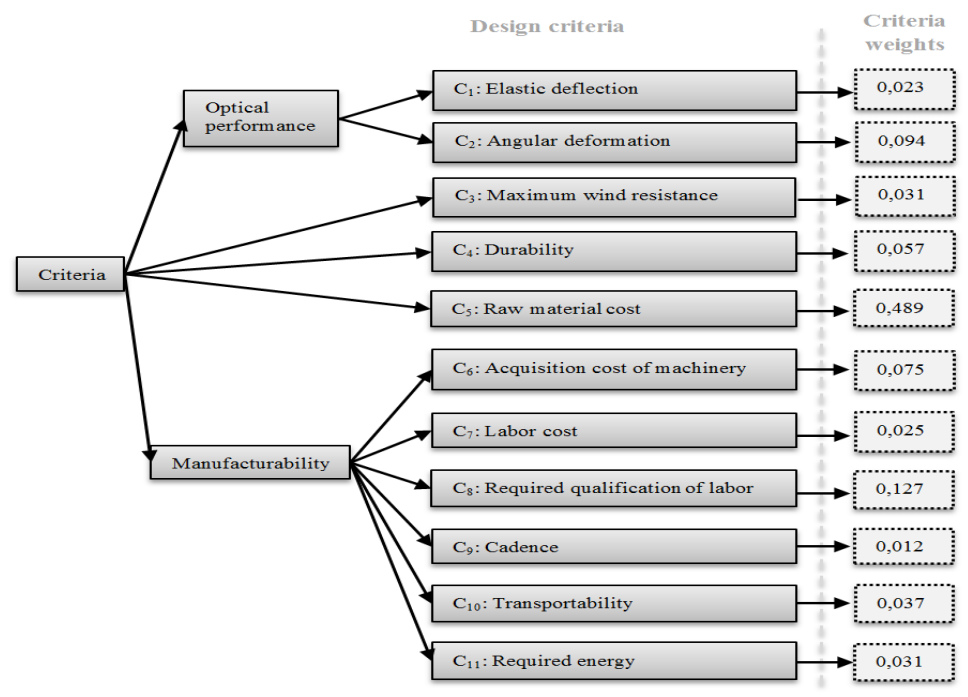

Figure 7 Design criteria with acceptance thresholds and weights

(Fig. 6). A mounting device is performed between the reflecting surface and the metal structure to ensure the connection between the two. Solar collector is driven by a rotation movement in order to track the movement of the sun. In our study, only the design of supporting structure (see Fig.6) is treated. The manufacturing of reflectors represents the main investment cost of a plant [31]. It is thus important to reduce the structure mass and also manufacturing cost. In addition, in order to maintain a good thermal efficiency of the plant, a high reflection performance is required from the collector. This implies that the elastic deformation of the structure must remain as low as possible. A high level of precision must also be considered when manufacturing the collector and fixing the reflecting surface. The entire criteria and there weights (obtained by AHP) are shown in Fig 7. However, acceptance thresholds cannot be shown because of confidentiality restrictions. 
Table 1 Observation variables

\begin{tabular}{lllllll}
\hline Design Concepts & TrS1 & TrS2 & TrS3 & RSS & TS1 & TS2 \\
\hline C1 & 4.31 & 4.21 & 4.21 & $\mathbf{1 9 . 7}$ & 12 & 11 \\
C2 & $\mathbf{0 . 0 3 1}$ & 0.028 & $\mathbf{0 . 0 3 3}$ & 0.015 & 0.011 & 0.011 \\
C3 & $\mathbf{6 8 7}$ & $\mathbf{6 5 4}$ & $\mathbf{7 5 1}$ & 1230 & 850 & 940 \\
C4 & 20 & 20 & 25 & 25 & 25 & $\mathbf{1 5}$ \\
C5 & 11.5 & 7.37 & 7.37 & 22 & 19.8 & 25 \\
C6 & 3 & 5 & 3 & 2.4 & 3.5 & 3.5 \\
C7 & $\mathbf{9 9}$ & $\mathbf{8 9}$ & 53 & 81 & 86 & 78 \\
C8 & $\mathbf{0 . 0}$ & 0.2 & $\mathbf{0 . 0}$ & 0.8 & $\mathbf{0 . 0}$ & $\mathbf{0 . 0}$ \\
C9 & 13 & 13 & 10 & 19 & 13 & 18 \\
C10 & 0.6 & 0.7 & 0.5 & 0.7 & $\mathbf{0 . 0}$ & $\mathbf{0 . 0}$ \\
C11 & 3 & $\mathbf{7}$ & 2 & 2.5 & 3.5 & 4 \\
\hline
\end{tabular}

A prior work has been performed within a design department developing a solar collector for CSP plant. It allows having enough knowledge of the product to use as a case study (observation variable, decision makers preferences, criteria relative priority). However, the data given in study case analysis will be limited due to confidentiality restrictions. As a result of the pre-dimensioning step, each concept is evaluated. It should be noted that the pre-dimensioning of manufacturing process is also performed during this phase to allow assessing of criteria related to manufacturability. Table 1 and 2 shows respectively the results of observation (dimensionless values) and interpretation steps. Concerning the aggregation function used to calculate OP metric. Equation 6 was used since none of proposed concepts respects the two conditions to be considered as a reference concept. The parameter s was fixed to 2 . The aggregation function becomes thus the quadratic mean (Equation 6). OP metric was first calculated using nominal values of IV (IV in Table 2). Imprecisions was then taken into account. Results of ASR and OP metrics (with estimated variations) are shown in Fig 8. Estimating the variations in $\mathrm{OP}$ values is important since it allows taking into account the impact of possible variations in the embodiment design choices made in the pre-dimensioned version (for example variations shown in Fig.4).

$$
O P=\sqrt{\sum_{j}^{n}\left(w_{j} I V_{j}^{2}\right)}
$$

As mentioned before, it is suitable in a product development project to have both mature/reliable concepts and innovative concepts with potentially better performance. However, in order to reduce risk, obtaining mature and reliable concept is a more pressing issue for decision makers. For this reason, RSS concept was chosen first to be developed. Its development began before the other concepts in the context of a previous project with many validation tests $(T R L \geq 6)$. The criterion of wind resistance is not satisfied due to the evolution of acceptance threshold in comparison with the previous requirements. However, the step of calculating the ASR metric elicits a high flexibility in 
Table 2 Interpretation variables

\begin{tabular}{lllllll}
\hline Design Concepts & TrS1 & TrS2 & TrS3 & RSS & TS1 & TS2 \\
\hline C1 & 0.8 & 0.8 & 0.8 & $\mathbf{0 . 0}$ & 0.5 & 0.5 \\
C2 & $\mathbf{0 . 0}$ & 0.3 & $\mathbf{0 . 0}$ & 0.7 & 0.7 & 0.6 \\
C3 & $\mathbf{0 . 0}$ & $\mathbf{0 . 0}$ & $\mathbf{0 . 0}$ & 0.3 & 0.7 & 0.6 \\
C4 & 0.5 & 0.5 & 0.7 & 0.7 & 0.7 & $\mathbf{0 . 0}$ \\
C5 & 0.7 & 0.9 & 0.9 & 0.4 & 0.3 & 0.2 \\
C6 & 0.3 & 0.1 & 0.8 & 0.7 & 0.6 & 0.6 \\
C7 & $\mathbf{0 . 0}$ & $\mathbf{0 . 0}$ & 0.7 & 0.4 & 0.3 & 0.4 \\
C8 & $\mathbf{0 . 0}$ & 0.2 & $\mathbf{0 . 0}$ & 0.8 & $\mathbf{0 . 0}$ & $\mathbf{0 . 0}$ \\
C9 & 0.7 & 0.7 & 0.7 & 0.9 & 0.7 & 0.9 \\
C10 & 0.6 & 0.7 & 0.5 & 0.7 & $\mathbf{0 . 0}$ & $\mathbf{0 . 0}$ \\
C11 & 0.7 & $\mathbf{0 . 0}$ & 0.8 & 0.7 & 0.5 & 0.6 \\
\hline
\end{tabular}

possible modifications of embodiment design features (addition of stiffeners, miner increase of structure height) to raise the wind resistance to the acceptance threshold. The ASR was thus approximated to 0.87 . The priority of design development is to continue the development of this concept until the desired maturity is reached. The equation (7) is then used in this case. The ASR and OP metrics are shown in Fig 8. TrS1 is a novel concept for the company and its low mass make it a very attractive concept because of the high weight of material cost criterion. Its OP metric is equal to 0.64 (the highest value). However, for this first pre dimensioned version, some of the criteria are not satisfied and thus many modifications should be done to satisfy all criteria. That explains the low ability to meet requirements obtained for this concept $A S R=0.25$. The third and later concept that was retained retain is TS1. It represents a good trade-off between OP and ASR metrics. The remaining concepts (rectangular tube, welted truss, hybrid truss) were abandoned because they present a low tradeoff between OP and ASR metrics. The budget allocated to product development is also a point to consider because it limits the number of concepts that can be developed in parallel. The obtained results for the two metric agree well with the expectations for this upstream phase in collector development process. As illustrated in Fig. 9, the choice of the three concepts by design department at the beginning of development project was well approved by the used metrics. It was remarked that the procedure of calculating ASR metric allows designers to easily elicit concepts with low opportunity of feasibility. As the product design is much driven by manufacturing process and this later has a low maturity. The main limit of the proposed methodology is the non-inclusion of manufacturing process maturity.

\section{Conclusion and future developments}

Decision making in conceptual engineering design have an important impact on overall life cycle cost. The need of supporting decision making by adequate theories and methodologies is thus greatest at this stage. This paper 


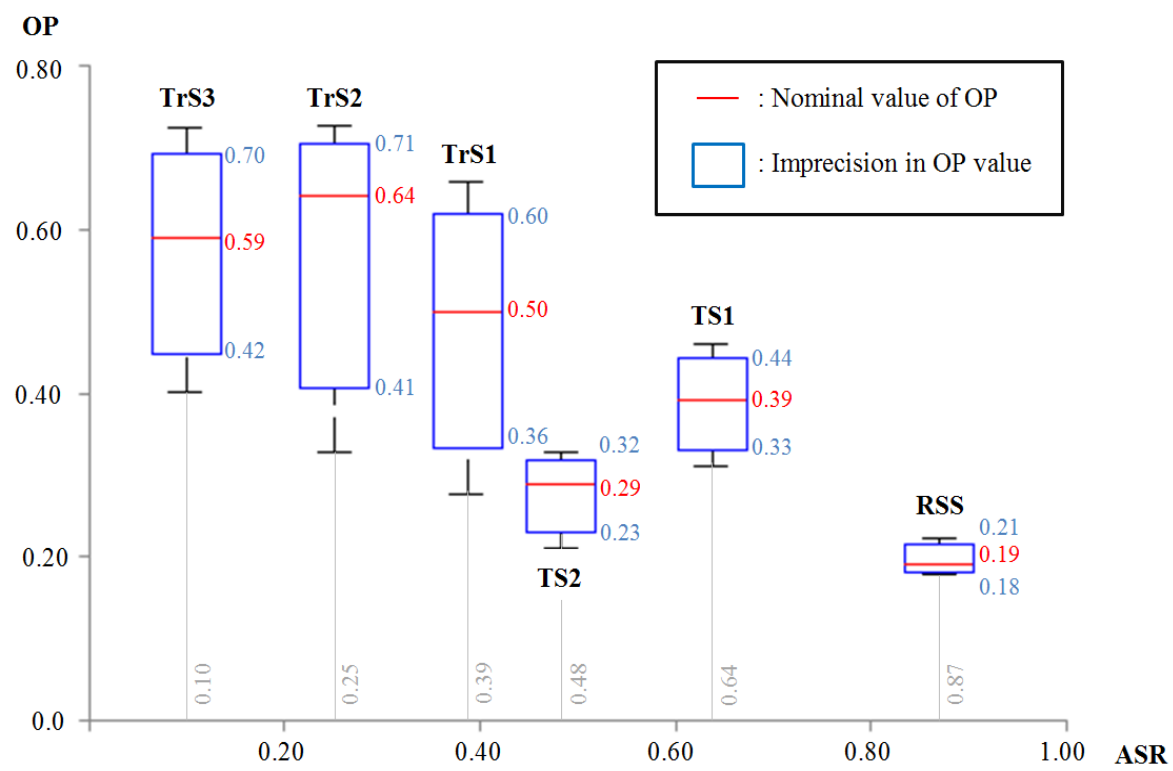

Figure 8 Results of OP and ASR metrics calculation for each concept

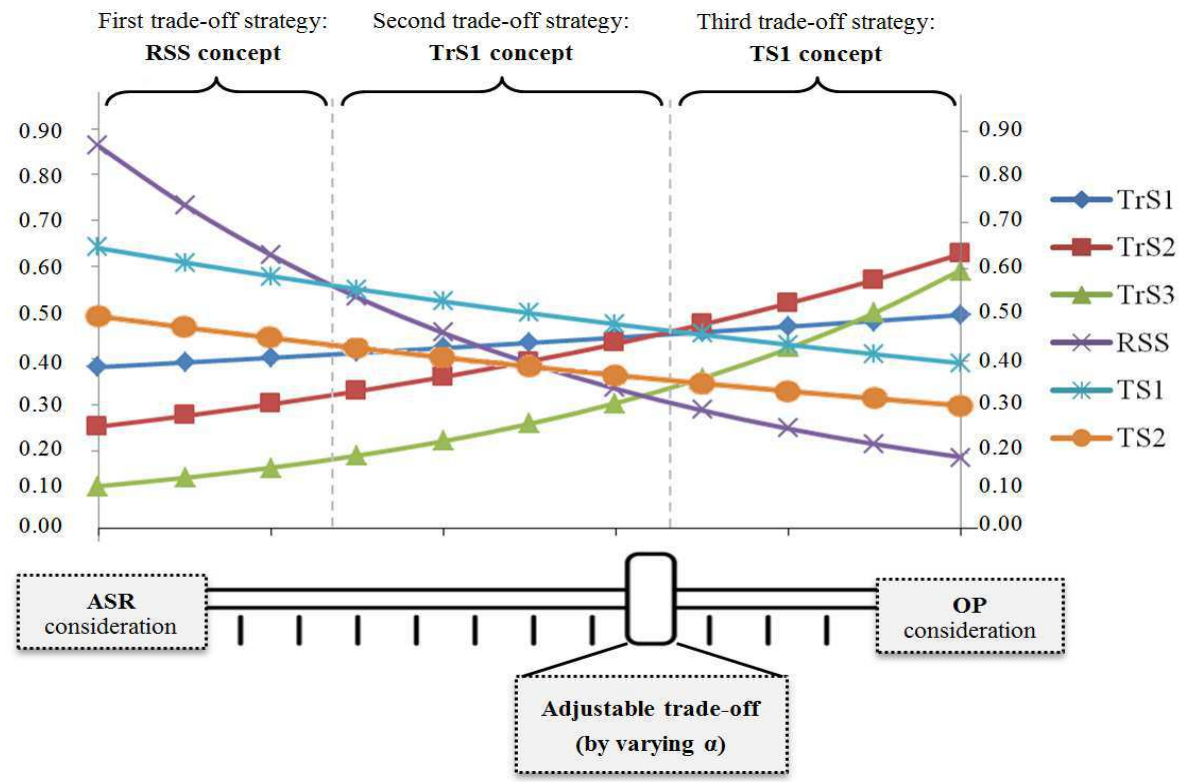

Figure 9 Ranking of the five concepts using different trade-off strategies between ASR and OP metrics 
present a general framework to carry out concepts screening based on the two main decision makers considerations which are the reduction of risk related to immature concepts and improvement of global performance. A first metric assess concepts ability to meet requirements and a second metric is proposed to assess concepts global performance. A parametric aggregation function was also proposed to support decision making using the two metrics. The main interest of these two metrics is to give a support for decision makers by allowing them to choose the set of concepts to develop with the desired trade-off between the confidence aspect (assessed by ASR metric) and the performance aspect (assessed by OP metric).

The obtained results for the two metric agree with the expectations for the early phase in collector development process. The choice of the three concepts by the design department at the beginning of development process was well approved by the used metrics. It was also remarked that the procedure of calculating ASR metric allows designers to easily elicit concepts with low opportunity of satisfying requirements and thus to prevent there development. However, the main limit of the proposed approach is the non-inclusion of manufacturing process maturity, especially for a context in which product design is highly driven by manufacturing process considerations. For further development, the integrating of manufacturing process maturity will be investigated. It is also planned to apply the proposed approach to another case studies to enhance its validity and eventually some other tools to allow its use in a more general context. Finally, more advanced stages in product development process will be investigated.

\section{Références}

1. Zimmer, L., Zablit, P. : Global aircraft predesign based on constraint propagation and interval analysis. Proceedings of CEAS Conference on Multidisciplinary Aircraft design and Optimisation. Kln, Germany (2001)

2. Morgan, J.M., Liker, J.K. : The Toyota Product Development System : Integrating People, Process and Technology. Productivity Press, Seattle (2006)

3. Sobek, D.K., Ward, A.C., Liker, J.K. : Toyotas Principles of Set-based Concurrent Engineering. Sloan Manage. Rev. 40(2), 6783 (1999)

4. Pahl, G., Beitz, W. : Engineering design A systematic approach, 2nd edn. SpringerVerlag, London (1996)

5. Ullman, D.G. : The Mechanical Design Process. McGraw Hill (2002)

6. O'Brien, C., Smith, S.J.E. : Design maturity assessment for concurrent engineering coordination. Int. J. Prod. Econ. 41(1-3), 311320 (1995)

7. Dunn, J. : Simultaneous engineering side-by-side to success. Engineer. 271(7020), 5153 (1990)

8. Beth, C.M., Konrad, M., Shrum, S. : CMMI for Development : Guidelines for Process Integration and Product Improvement, 3rd edn. Addison-Wesley (2007)

9. Katz, D.R., Sarkani, S., Mazzuchi, T., Conrow, E.H. : The Relationship of Technology and Design Maturity to DoD Weapon System Cost Change and Schedule Change During Engineering and Manufacturing Development. Syst. Eng. 18(1), 115 (2014) 
10. Sharif, N., Huang, C. : Innovation strategy, firm survival and relocation : The case of Hong Kong-owned manufacturing in Guangdong Province. China. Res. Policy 41, 6978 (2012)

11. Guitouni, A., Martel, J.M. : Tentative guidelines to help choosing an appropriate MCDA method. Eur. J. Oper. Res. 109, 501521 (1998)

12. Collignan, A., Sebastian, P., Pailhes, J., Ledoux, Y. : Arc-elasticity and hierarchical exploration of the neighborhood of solutions in mechanical design. Adv. Eng. Inform. 26, 603617 (2012)

13. Wood, K.L., Antonsson, E.K. : Computations with imprecise parameters in engineering design : background and theory. J. Mech. Transm-T ASME. 111(4), 616625 (1989)

14. Zadeh, L.A. : Fuzzy Sets. Inform. Control. 8, 338353 (1965)

15. Okudan, G.E., Tauhid, S. : Concept selection methods a literature review from 1980 to 2008. Int. J. Des. Eng. 1(3), 243277 (2008)

16. El Amine, M., Pailhs, J., Perry, N. : Selection and use of a multi-criteria decision aiding method in the context of conceptual design with imprecise information : Application to a solar collector development. Concurrent Eng-Res. A. 24(1), 3547 (2016)

17. Roy, B., Slowinski, R. : Questions guiding the choice of a multicriteria decision aiding method. Eur. J. Decis. Process. 1, 6997 (2013)

18. Neumann, J.V., Morgenstern, O. : Theory of games and economic behavior. In : Wiley, J. (eds.), pp. 1013. Science editions (1944)

19. Pahl, G., Beitz, W. : Engineering Design. In : The Design Council, pp.119138. London (1984)

20. Scott, M.J., Antonsson, E.K. Aggregation functions for engineering design trade-offs. Fuzzy Set Syst. 99(3), 253264 (1998)

21. Malak, Jr. R.J., Aughenbaugh, J.M., Paredis, C.J.J. : Multi-attribute utility analysis in set-based conceptual design. Comp. Aid. Des. 41(3), 214227 (2009) 